ORIGINAL ARTICLE

\title{
Frequency of Different Types of Arthritis in patients of Psoriasis
} \author{
BUKHARI $^{4}$, UZMA SARWAR ${ }^{5}$, HAFIZ BASHIR AHMED KALHORO ${ }^{6}$ \\ ${ }^{1}$ Senior Registrar, Department of Dermatology, Shalamar Medical \& Dental College Hospital, Lahore \\ ${ }^{2}$ Professor and HOD, Department of Dermatology, Services Hospital, Lahore. \\ ${ }^{3}$ Senior Registrar, Fatima Memorial Hospital, Shadman, Lahore. \\ ${ }^{4}$ Professor, Department of Dermatology, Shalamar Medical \& Dental college Hospital, Lahore \\ ${ }^{5}$ Associate Professor, Department of Dermatology, Shalamar Medical \& Dental college Hospital, Lahore \\ ${ }^{6}$ Assistant Professor, Department of Dermatology, Liaquat University Hospital, Hyderabad \\ Correspondence to Dr. Aijaz Zeeshan Khan ChacharEmail: dr_aijaz84@hotmail.com, Cell: 0333-2612096
}

ANNUM ASHRAF ${ }^{1}$, MUHAMMAD SHAHBAZ AMAN ${ }^{2}$, AIJAZ ZEESHAN KHAN CHACHAR ${ }^{3}$, MUHAMMAD AZAM

\begin{abstract}
Background: Psoriatic arthritis (PsA) is type of inflammatory arthritis associated with psoriasis- There are multiple patterns of joint involvement in patients with psoriasis

Aim: To determine the frequency of arthritis and its different types in patients of psoriasis presenting at a tertiary care hospital in Lahore

Methods: This cross Sectional study was conducted in Dermatology Department of Services Hospital, Lahore from 11th April, 2017 to $10^{\text {th }}$ October, 2017. Two hundred and sixty patients having psoriasis were enrolled in the study, Patients' detailed history about psoriasis, duration of psoriasis and joint pain or stiffness was taken. After written and informed consent Physical examination was done to determine the type of psoriasis and nail involvement. All the joints were examined for tenderness and deformities to assess the evidence of arthritis and its different types according to the site of involved joints.

Results: Total numbers of patients included in the study was 260; in which $176(67.7 \%)$ patients were males and $84(32.3 \%)$ were females with the total mean age of $38 \pm 13.22$ years. Out of 260 patients, $62(23.8 \%)$ patients had psoriatic arthritis (PsA), Out of these 62 patients having PsA, 23(37.1\%) patients had Oligoarthritis, followed by Symmetric arthritis 19(30.6\%) patients, Distal interphalangeal arthritis (DIP) in $15(24.2 \%)$.

Conclusion: Our study concluded that almost one fourth of psoriasis patients had arthritis, asymmetrical oligoarthritis/monoarthritis came out to be the most common type in our study followed by symmetric polyarthritis, distal interphalangeal arthritis (DIP), Arthritis mutilans and axial arthritis

Keywords: Psoriasis, types of psoriasis, arthritis, psoriatic arthropathy
\end{abstract}

\section{INTRODUCTION}

Psoriasis is a chronic, inflammatory and proliferative condition of skin in which both genetic and environmental factors play a vital role. People are considered to have psoriasis if they have characteristic lesions consisting of red, scaly, sharply demarcated, indurated plaques, present particularly over scalp and extensor surfaces of the body. The disease is enormously variable in duration, periodicity of flares and extent with different morphological types. ${ }^{1}$ the prevalence of psoriasis in general population is 1$2 \% .{ }^{2}$ Psoriasis occurs in men and women equally ${ }^{3}$, and is reported in all races. Although psoriasis can start at any age, there are two peaks in its occurrence early onset Psoriasis which occur between ages 20 and 30 and late onset psoriasis which occurs between ages 50 and $60 .{ }^{4}$

Psoriatic arthritis is type of inflammatory arthritis associated with psoriasis. ${ }^{5}$ earlier it was postulated that psoriatic arthritis is a clinical variant of rheumatoid arthritis, but as the research progressed and it has proven that psoriatic arthritis is a separate clinical entity. ${ }^{6}$

Most of the experts have consensus upon Classification Criteria for Psoriatic Arthritis (CASPAR) criteria, first time launched in 2006 and using this criteria for the first time revealed that estimated prevalence of PsA in patients having psoriasis in two large general practices in the United Kingdom was $14 \%{ }^{7}$. A prospective four-

Received on 27-02-2021

Accepted on 26-05-2021 year study of patients with psoriasis who did not have psoriatic arthritis at diagnosis of psoriasis revealed an incidence of newly diagnosed psoriatic arthritis, using the CASPAR criteria was 2 percent per year. ${ }^{8}$ The severity of psoriasis, the presence of nail lesions, and the presence of scalp and intergluteal lesions were strongly associated with psoriatic arthritis and more observed in these patients.In one prospective study of 1018 patients with early synovitis, a diagnosis of psoriatic arthritis was made in 129 patients which is equal to (13\%), out of these patients (6 percent) did not have any signs of psoriasis at presentation. ${ }^{9}$

The most frequent presentations are polyarthritis, followed by oligoarthritis. Distal involvement alone occurs in less than 20 percent of cases and may be associated with spondyloarthritis. On the other hand, arthritis mutilans can occur with any of these patterns. So we wanted to highlight and find out the frequency of arthritis and its different types in patients of psoriasis presenting at a tertiary care hospital in Lahore.

\section{PATIENTS AND METHODS}

This cross Sectional study was conducted in Dermatology Department of Services Hospital, Lahore for six months after approval from ethical committee. Sampling technique used was non probability consecutive sampling. Sample size of 260 patients was calculated with $95 \%$ confidence level $6 \%$ margin of error and taking average prevalence of PsA i.e. $40 \%$ in patients with Psoriasis. ${ }^{10}$ 


\section{Inclusion criteria}

- Age Patients of age groups (20-60 years)

- Gender (Male/Female)

- Diagnosed patients of Psoriasis (clinical or histopathology if needed)

\section{Exclusion criteria}

- All patients having any other connective tissue disease (diagnosed clinically and serological analysis of Antinuclear antibodies(ANA), extractable nuclear antigen (eNA), Anti-cyclic citrullinated peptide (antiCCP) antibodies if required)

- All Patients having concomitant other forms of arthritis (osteoarthritis, spondyloarthritis, Reiters disease etc diagnosed clinically and joint fluid analysis if required)

- All patients having Diabetes Mellitus (according to fasting blood sugar levels)

- All patients having acute bacterial, viral or fungal infections which causes septic arthritis (diagnosed clinically and joint fluid analysis if required)

- All patients having human immunodeficiency virus(HIV) and Syphilis (history and HIV serology, venereal disease research laboratory(VDRL) test, Treponema pallidum hemagglutination (TPHA) analysis)

- All patients having any form of drug reactions (history of recent drug intake)

Data collection procedure: The study was conducted in department of Dermatology Services Hospital Lahore. 260 patients fulfilling the inclusion/exclusion criteria were enrolled. Written and Informed consent was taken from patients. Name, age, gender \& address were noted. Patients' detailed history about psoriasis, duration of psoriasis and joint pain or stiffness was taken. Physical examination was done to determine the type of psoriasis and nail involvement. All the joints were examined for tenderness and deformities to assess the evidence of arthritis and its different types according to the site of involved joints. Findings were confirmed by a consultant of the Department of Dermatology having vast experience in dealing with such kind of patients to avoid any bias for diagnosis of different forms of psoriatic arthritis.

$X$-rays of the involved joints were done to confirm the arthritis and its different types. Radiological changes were noted. This information was noted on proforma

Statistical analysis: Collected data was entered and analyzed in SPSS version 23.0 Mean \pm standard deviation were calculated for quantitative variable like age and duration of psoriasis. Frequencies and percentages were calculated for qualitative variables like sex, arthritis and its different types in psoriasis patients. Stratification was done with respect to age, gender, nail involvement, duration of disease and type of psoriasis. Frequency of arthritis and its different types were the outcome variables. Chi-square test was applied post stratification considering $p$-value $\leq 0.05$ as significant.

\section{RESULTS}

Total number of patients included in the study was 260; in which 176 (67.7\%) patients were males and 84 (32.3\%) were females with total mean age of $38 \pm 13.226$ years. Out of 260 patients, $62(23.8 \%)$ patients had psoriatic arthritis
(PsA), out of which $44(16.9 \%)$ were males and $18(6.9 \%)$ were females. Out of these 62 patients having PsA, $23(37.1 \%)$ patients had Oligoarthritis, followed by Symmetric arthritis $19(30.6 \%)$ patients, Distal interphalangeal arthritis (DIP) in 15 (24.2\%), Arthritis mutilans in 3(4.8\%) and Axial arthritis in 2(3.2\%).

With the duration of psoriasis from more than 1 year to 5 years, $10(3.8 \%)$ patients had PsA, patients having psoriasis from 6 to 10 years, 34 (13.1\%) patients had PsA, patients having psoriasis of more than 10 years, $18(6.9 \%)$ patients were found to have PsA. $(p-v a l u e=0.0001)$

Out of 62 patients with PsA, 10(16.1\%) fell in the age category of 20 to 30 years, $5(18.1 \%)$ patients were in age category of $31-40$ years, $13(20.9 \%)$ patients were in age category of $41-50$ years and $34(54.8 \%)$ patients were age category of 51-60 years.

Out of these 62 patients, $52(83.81 \%)$ had plaque psoriasis, 4(6.4\%) patients had guttate psoriasis, $5(8.1 \%)$ patients had pustular psoriasis and 1 (1.6\%) patient had scalp psoriasis. Out of these 62 patients with PsA, $54(87 \%)$ patients had nail changes8 (13\%) patients with PsA did not have nail changes ( $p$-value $=0.0001$ ).

PsA was stratified with respect to age and gender, there was significant association with regard to age and frequency of arthritis but no statistical association was found with regard to gender. Nail involvement and plaque type psoriasis was found to be statistically significant with PsA. $(p$-value $=0.0001)$

Table I: Frequency of types of Psoriasis with regard to Psoriatic arthritis

\begin{tabular}{|l|c|c|c|}
\hline $\begin{array}{l}\text { Type of } \\
\text { Psoriasis }\end{array}$ & $\begin{array}{c}\text { Psoriatic arthritis } \\
\text { present }\end{array}$ & $\begin{array}{c}\text { Psoriatic } \\
\text { arthritis absent }\end{array}$ & Total \\
\hline Plaque & $52(20 \%)$ & $138(53 \%)$ & $190(73.1 \%)$ \\
\hline Guttate & $4(1.5 \%)$ & $20(7.7 \%)$ & $24(9.2 \%)$ \\
\hline Pustular & $5(1.9 \%)$ & $16(6.2 \%)$ & $21(8.2 \%)$ \\
\hline Scalp & $1(0.4 \%)$ & $23(8.8 \%)$ & $24(9.2 \%)$ \\
\hline Erythrodermic & $0(0 \%)$ & $1(0.4 \%)$ & $1(0.4 \%)$ \\
\hline Total & $62(23.8 \%)$ & $198(76.2 \%)$ & $260(100 \%)$ \\
\hline
\end{tabular}

Table II: Frequency of different types of arthritis in Psoriatic arthritis

\begin{tabular}{|l|c|c|}
\hline Type of Psoriatic arthritis & n & $\%$ age \\
\hline Peripheral Mono or asymmetric Oligoarthritis & 23 & 37.1 \\
\hline Symmetric Polyarthritis & 19 & 30.6 \\
\hline Distal Interphalangeal arthritis (DIP) & 15 & 24.2 \\
\hline Arthritis Mutilans & 3 & 4.8 \\
\hline Axial arthritis & 2 & 3.2 \\
\hline Total & 62 & 100 \\
\hline
\end{tabular}

Table III: Frequency of Psoriatic arthritis and its types

\begin{tabular}{|l|c|c|}
\hline Type of Psoriatic arthritis & $\mathbf{n}$ & \%age \\
\hline Peripheral Mono or asymmetric Oligoarthritis & 23 & 8.8 \\
\hline Symmetric Polyarthritis & 19 & 7.3 \\
\hline Distal Interphalangeal arthritis (DIP) & 15 & 5.8 \\
\hline Arthritis Mutilans & 3 & 1.2 \\
\hline Axial arthritis & 2 & 0.8 \\
\hline No arthritis & 198 & $76.2 \%$ \\
\hline Total & 260 & 100 \\
\hline
\end{tabular}

Table: IV: Frequency of PsA

\begin{tabular}{|l|c|c|}
\hline $\begin{array}{l}\text { Psoriatic arthritis } \\
\text { present }\end{array}$ & Psoriatic arthritis absent & Total \\
\hline 0 & $28(10.8 \%)$ & $28(10.8 \%)$ \\
\hline $10(3.8 \%)$ & $130(50 \%)$ & $140(53.8 \%)$ \\
\hline $34(13.1 \%)$ & $39(15 \%)$ & $73(28.1 \%)$ \\
\hline $18(6.9 \%)$ & $1(0.4 \%)$ & $19(7.3 \%)$ \\
\hline $62(23.8 \%)$ & $198(76.2 \%)$ & $260(100 \%)$ \\
\hline
\end{tabular}


Graph I: Association of Different types of psoriasis with psoriatic arthritis



Graph. II: Different types of Psoriasis with Psoriatic arthritis

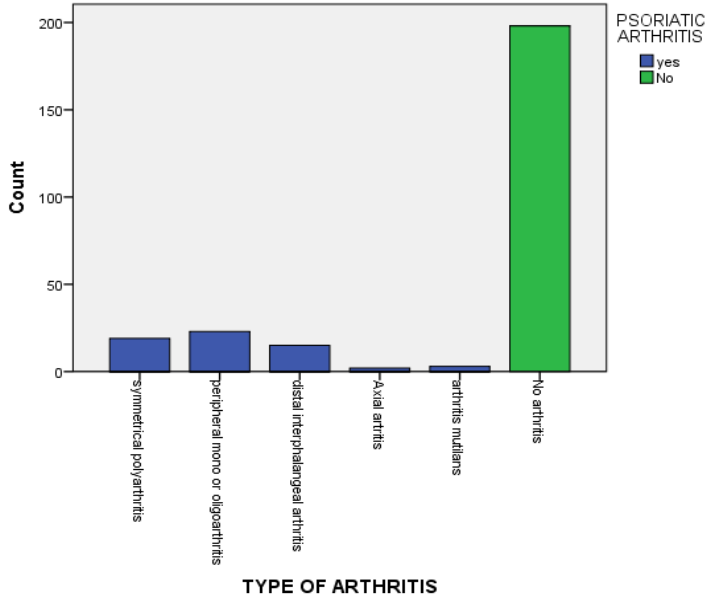

\section{DISCUSSION}

Psoriasis is a chronic, inflammatory disease with a prevalence of $2-3 \%$ in the world population. It is associated with many other co-morbidities like cardiovascular diseases and hence the new term is being used frequently known as psoriatic disease to signify the different co-morbidities associated with the patients having psoriasis. ${ }^{11}$ Approximately $30 \%$ of the patients with psoriasis go on to develop and suffer from psoriatic arthritis. ${ }^{12}$ Psoriasis occurred before arthritis in $68 \%$ of patients, occurred simultaneously in $11 \%$ of the patients and followed it in $21 \%$ patients. ${ }^{1}$ The aim of this study was to determine the frequency of PsA in patients with psoriasis using these new validated diagnostic criteria and its different forms in various types of psoriasis in our community. Recent studies have demonstrated an increased risk of essential hypertension, ischemic heart disease, and stroke in psoriatic patients.

Total number of patients in our study were 260 and study conducted by Reich K, et al..$^{13}$ enrolled 1511 patients, this significant difference among number of patients enrolled can be explained as study conducted by Reich K, et al. ${ }^{13}$ was multicentred from 48 centres and of long duration as compared to our study which was a single center study with duration of six months.

In our study, out of 260 patients, 176 (67.7\%) were males and $84(32.3 \%)$ were females where as in a study conducted by Ejaz et al, ${ }^{14}$ out of 100 patients, $71 \%$ were males and and $29 \%$ were females which is comparable with our study as in our setup, male patients report earlier as compared to females.

All of the patients included in our study were between 20-60 years of age as most of the studies conducted also had the same age group, like the study conducted by Siddiqui S, et al. ${ }^{15}$ age limitation was $18-65$ years of age which is comparable with our study as most of the patients of psoriasis present after early adolescence.

Our study revealed that $23.8 \%$ patients had PsA, and a study conducted in Germany by Reich K, et al. ${ }^{13}$ concluded that prevalence of PsA was $20.6 \%$ which again reflects that the results are comparable with our study as one third of the patients of psoriasis develop PsA globally at some point of their age.

A recent local study conducted at civil hospital, Karachi by Siddiqui S, et al. ${ }^{15}$ revealed prevalence of PsA as $39.5 \%$ in local population and our study showed $23.8 \%$, this minor difference can be attributed as our study had bigger sample size than this study.

Our study showed $16.9 \%$ male patients had PsA and female patients only had $6.9 \%$ prevalence of PsA and study conducted by Trontzas $P$, et al. ${ }^{16}$ revealed male predominance in PsA which also matches with our study. This can also be attributed to as more male patients attending in our hospital.

Our Study showed that most of the PsA patients presented in their second and fifth decade of life (60.4\%) collectively and Studies from the India also show the onset in the $3 \mathrm{rd}$ and 4 th decades of life $(70 \%),{ }^{12}$ which is also comparable with our study as around $68 \%$ of patients develop arthritis after psoriasis which is a chronic inflammatory process. ${ }^{1}$

Our Study showed that Peripheral/ Monoarthritis or Oligoarthritis was the most common type of arthritis in patients with PsA, 37\% patients had Peripheral/ Monoarthritis Oligioarthritis and $30.6 \%$ patients had Symmetric polyarthritis where as Sitwat Siddiqui, et al. ${ }^{15}$ concluded that amongst types of PsA that Peripheral/ Monoarthritis or Oligoarthritis was found in $37 \%$ of the patients with PsA and 34\% patients were found to suffer from symmetric polyarthritis, Similarly, a study conducted by Ejaz A, et al. ${ }^{14}$ revealed that $34.7 \%$ had Peripheral Monoarthritis or Oligoarthritis and $30.6 \%$ patients had Symmetric polyarthritis. These results are highly comparable with our study as Peripheral mono or asymmetrical oligo arthritis is most frequently presenting type of arthritis in patients of psoriasis worldwide.

A study conducted in India by Kumar R, et al. 12 concluded that $21 \%$ of their patients with PsA had 
Peripheral Monoarthritis or Oligoarthritis, our study showed $37.1 \%$ had Oligoarthritis, these differences can be attributed as Study conducted by Kumar R, et al. had four times larger sample size as compared to ours.

Our study showed $24.2 \%$ of PsA patients had Distal Interphalangeal arthritis and $3.2 \%$ patients had Axial arthritis and study conducted in Kashmir by Rather $S$, et al. ${ }^{17}$ revealed that Distal Interphalangeal arthritis was found in $8 \%$ and Axial arthritis in $6 \%$ of the patients with PsA. This difference of study can be attributed to the small population size of study conducted by Rather S, et al. Less frequency of axial arthritis can be explained by the fact that axial arthritis usually co exists with other common forms of arthritis and can be overlooked.

Arthritis mutilans was found in $3(1.2 \%)$ patients in our study and similarly arthritis mutilans was judged as the least common clinical type of PsA which is clinically seen in only about $1 \%$ of Indian patients. ${ }^{12}$ and $2 \%$ in a study conducted by Rather $\mathrm{S}$ et al. ${ }^{17}$ both results are comparable with our study. These patients present with severe, progressive inflammation of joints which ultimately results in the destruction of the joints leading to a permanent deformity and disability.

A study conducted in Greece in 2016 by Papadavid E, et al. ${ }^{18}$ revealed that $86 \%$ of the all patients with PsA had plaque type of psoriasis which is comparable with our study in which $83.8 \%$ of PsA patients had plaque psoriasis. This can be explained by the fact that plaque psoriasis is most common form of psoriasis and runs a more chronic course as compared to other subtypes.

Our Study concluded that $87 \%$ of the patients with PsA had nail involvement and a study conducted by Ejaz A, et $\mathrm{al}^{14}$ found that nail involvement was found in $74 \%$ of the patients with PsA. Also a study conducted by Rather S, et al. ${ }^{17}$ found that nail involvement was found in $80 \%$ of the patients with PsA which can easily be compared with our study because the nail involvement like pitting, ridging, subungual hyperkeratosis etc is the most frequent manifestation of psoriasis and especially in patients of arthritis involving hands and feet. Hence nail changes in a patient of pasoriasis should prompt to investigate for arthritis.

\section{CONCLUSION}

Psoriatic arthritis (PsA) is not uncommon and found in about one fourth of psoriasis patients. Our study concluded that among different clinical types of arthritis in patients with psoriasis are asymmetrical oligoarthritis/monoarthritis, most commonly observed in our study followed by symmetric polyarthritis, distal interphalangeal arthritis (DIP), axial arthritis and Arthritis mutilans.

Limitations: Our study was single centre with relatively smaller sample size, so results cannot be generalized to whole population.

Recommendations: Psoriasis is a disease with no complete cure and it greatly affects the quality of life so further multicentre studies with larger sample size are still required to relieve from early disability and misery. It is the need of the day to create awareness among general population and for general practioners through electronic media, print media and arranging seminars and symposia about the disease and its early diagnosis and so as to prevent the complications related with this disease and by doing so reducing the misery and early disability of the patients and improving the quality of life.

Contribution: AA: Conception of the study, Data collection, Review of literature, Article writing, Review of manuscript, MSA: Conception of the study, Data Interpretation, Review of literature, Review of Manuscript, Final approval of Manuscrip, AZKC: Development of Research Methodology Design, Study Design, Article writing, Review of Literature, Data analysis \& Data Interpretation, Drafting article, MAB: Data Interpretation, Review of literature, Review of Manuscript, Final approval of Manuscript, US: Review of literature, Review of Manuscript, Drafting Article, HBAK: Review of literature, Review of Manuscript, Drafting Article

\section{REFERENCES}

1. Burden A, Kirby B. Psoriasis and Related Disorders. In Griffiths C.E.M, Barker J, Bleiker T, Chalmers R, Creamer D, ed. By. Rook's Text book of Dermatology. $9^{\text {th }}$ ed. United Kingdom John Wiley \& Sons, Limited 2016. P. 35.1-32.

2. Brent LH. Inflammatory arthritis an overview for primary care Physicians. Postgrad Med 2009; 121:148-62.

3. Gelfand JM, Weinstein R, Porter SB, et al. Prevalence and treatment of psoriasis in the United Kingdom a population-based study. Arch Dermatol 2005; 141:1537.

4. Farber EM, Nall ML. The natural history of psoriasis in 5,600 patients. Dermatologica $1974 ; 1481$.

5. Brockbank J, Gladman D. Diagnosis and management of psoriatic arthritis. Drugs 2002; 62:2447.

6. Wright V, Moll JM. Psoriatic arthritis. Bull Rheum Dis 1971; 21:627.

7. Ibrahim G, Waxman R, Helliwell PS. The prevalence of psoriatic arthritis in people with psoriasis. Arthritis Rheum 2009; 61:1373.

8. Eder L, Chandran V, Shen $\mathrm{H}$, et al. Incidence of arthritis in a prospective cohort of psoriasis patients. Arthritis Care Res (Hoboken) $2011 ; 63: 619$

9. Kane D, Stafford L, Bresnihan B, FitzGerald O. A prospective, clinical and radiological study of early psoriatic arthritis an early synovitis clinic experience. Rheumatology (Oxford) 2003; 42:1460.

10. Christophers E, Barker JN, Griffiths CE. The risk of psoriatic arthritis remains constant following initial diagnosis of psoriasis among patients seen in European dermatology clinics. J Eur Acad Dermatol Venereol 2010; 24:548.

11. Ritchlin C. Psoriatic disease - from skin to bone. Nat Clin Pract Rheumatol 2007; 3:698-706.

12. Kumar R, Sharma A, Dogra S. Prevalence and clinical patterns of psoriatic arthritis in Indian patients with psoriasis. Indian J Dermatol. 2014; 80:15-23.

13. Reich K, Krüger K, Mössner R, Augustin M. Epidemiology and clinical pattern of psoriatic arthritis in Germany a prospective interdisciplinary epidemiological study of 1511 patients with plaque-type psoriasis. $\mathrm{Br} \mathrm{J}$ Dermatol. 2009; 160(5):1040-7.

14. Ejaz A, Iftikhar A, Iftikhar N. Patterns of Psoriatic Arthritis. Journal of the College of Physicians and Surgeons Pakistan 2009; 19 (9):553-56.

15. Sitwat Siddiqui, Zarnaz Wahid, Humaira Talat. Frequency and clinical patterns of psoriatic arthritis in patients of psoriasis. Journal of Pakistan Association of Dermatologists. 2016; 26 (4):328-331.

16. Trontzas $\mathrm{P}$, Andrianakos $\mathrm{A}$, Miyakis $\mathrm{S}$ et al. Seronegative spondyloarthropathies in Greece a population-based study of prevalence, clinical pattern, and management. The ESORDIG study. Clin. Rheumatol. 2005.24(6):583-589.

17. Rather S, Nisa N, Arif T. The Pattern of Psoriatic Arthritis in Kashmir A 6-Year Prospective Study. N Am J Med Sci. 2015 Aug; 7(8): 356-361.

18. Papadavid E, P. Katsimbri,I. Kapniari,D. Koumaki,A. Karamparpa, et al.Prevalence of psoriatic arthritis and its correlates among patients with psoriasis in Greece results from a large retrospective study. European Academy of Dermatology and Venereology. 2016 Oct; 30(10):1749-1752 